\title{
Orientation Selectivity of Synaptic Input to Neurons in Mouse and Cat Primary Visual Cortex
}

\author{
Andrew Y. Y. Tan (陈勇毅), Brandon D. Brown, Benjamin Scholl, Deepankar Mohanty, and Nicholas J. Priebe \\ Center for Perceptual Systems, Section of Neurobiology, School of Biological Sciences, College of Natural Sciences, The University of Texas at Austin, \\ Austin, Texas 78705
}

Primary visual cortex (V1) is the site at which orientation selectivity emerges in mammals: visual thalamus afferents to V1 respond equally to all stimulus orientations, whereas their target V1 neurons respond selectively to stimulus orientation. The emergence of orientation selectivity in $\mathrm{V} 1$ has long served as a model for investigating cortical computation. Recent evidence for orientation selectivity in mouse V1 opens cortical computation to dissection by genetic and imaging tools, but also raises two essential questions: (1) How does orientation selectivity in mouse V1 neurons compare with that in previously described species? (2) What is the synaptic basis for orientation selectivity in mouse V1? A comparison of orientation selectivity in mouse and in cat, where such measures have traditionally been made, reveals that orientation selectivity in mouse V1 is weaker than in cat V1, but that spike threshold plays a similar role in narrowing selectivity between membrane potential and spike rate. To uncover the synaptic basis for orientation selectivity, we made whole-cell recordings in vivo from mouse V1 neurons, comparing neuronal input selectivity—based on membrane potential, synaptic excitation, and synaptic inhibition - to output selectivity based on spiking. We found that a neuron's excitatory and inhibitory inputs are selective for the same stimulus orientations as is its membrane potential response, and that inhibitory selectivity is not broader than excitatory selectivity. Inhibition has different dynamics than excitation, adapting more rapidly. In neurons with temporally modulated responses, the timing of excitation and inhibition was different in mice and cats.

\section{Introduction}

Neuronal response selectivity for stimulus orientation is one of the properties that mouse V1 shares with its mammalian counterparts (Dräger, 1975; Mangini and Pearlman, 1980; Métin et al., 1988; Hübener, 2003; Niell and Stryker, 2008; Gao et al., 2010). In mammals, neurons in the visual thalamus - the afferents to V1are selective for specific spatial locations, but respond equally to all stimulus orientations within the preferred spatial location. Neurons in V1, on the other hand, are selective for stimulus orientation, marking the emergence of this response property in the visual pathway (Hubel and Wiesel, 1962). The underlying synaptic basis for this orientation tuning may provide insight into what computations are occurring at the level of cortex to generate such transformations (Sompolinsky and Shapley, 1997). While the generation of orientation selectivity in V1 has been well studied in several mammalian species, it is poorly understood in the mouse (Ferster and Miller, 2000; Shapley et al., 2003; Hirsch and Martinez, 2006; Alitto and Dan, 2010). To use recent advances in genetic and imaging technologies available in mice, it is essential to understand the computations occurring in mouse V1. Here we

Received April 24, 2011; revised July 4, 2011; accepted July 12, 2011.

Author contributions: A.Y.Y.T., B.D.B., and N.J.P. designed research; A.Y.Y.T., B.D.B., B.S., D.M., and N.J.P. performed research; A.Y.Y.T., B.D.B., and N.J.P. analyzed data; A.Y.Y.T., B.D.B., and N.J.P. wrote the paper.

This work was supported by grants from the National Institutes of Health (EY-019288) and the Pew Charitable Trusts. We are grateful to Jessica Hanover and David Ferster for helpful discussions.

Correspondence should be addressed to Nicholas J. Priebe, Center for Perceptual Systems, Section of Neurobiology, The University of Texas at Austin, 2400 Speedway, Austin, TX 78705. E-mail: nicholas@mail.utexas.edu.

DOI:10.1523/JNEUROSCI.2039-11.2011

Copyright $\odot 2011$ the authors $\quad 0270-6474 / 11 / 3112339-12 \$ 15.00 / 0$ describe and compare the synaptic basis for orientation selectivity in mouse and cat $\mathrm{V} 1$, and relate our findings to existing models of cortical computation.

One proposed model for the generation of cortical orientation selectivity is that incoming excitation provides a bias for selectivity, which inhibition sculpts by suppressing responses at nonpreferred orientations. Consistent with this model, inhibitory neurons in mouse V1 are weakly selective for orientation compared to excitatory neurons (Gonchar et al., 2007; Sohya et al., 2007; Niell and Stryker, 2008). An alternative model is that orientation selectivity emerges from the convergence of similarly tuned excitatory and inhibitory input, with increased orientation selectivity emerging via the spike threshold nonlinearity (Priebe and Ferster, 2008; Jia et al., 2010). The first hypothesis predicts a higher selectivity for excitatory input relative to inhibitory input, whereas the second hypothesis predicts equal degrees of selectivity for both classes of synaptic inputs. Furthermore, it is unclear whether excitatory and inhibitory inputs have similar or distinct orientations preferences in mouse V1, and whether they are coincident in time.

We therefore examined the synaptic excitation and inhibition underlying orientation selectivity using whole-cell recordings in vivo. We estimated excitation and inhibition using both currentand voltage-clamp recordings. Neurons that received highly selective excitatory inputs also received highly selective inhibitory inputs. Neurons that received weakly selective excitatory inputs also received weakly selective inhibitory inputs. Orientation selectivity of both excitatory and inhibitory inputs was highly correlated to membrane potential selectivity. Therefore, as in other 
sensory modalities and as in cat V1, computations in mouse V1 use overall shared excitatory and inhibitory selectivity, instead of differences in excitatory and inhibitory selectivity. A direct comparison between cat and mouse reveals differences in the degree of orientation selectivity, but similar connectivity rules generating selectivity.

\section{Materials and Methods}

Physiology. Physiological procedures were based on those previously described (Gordon and Stryker, 1996; Hanover et al., 1999). We used postcritical period C57BL/6 mice of age 5-12 weeks. Mice were anesthetized with intraperitoneal injection of $50-80 \mathrm{mg} / \mathrm{kg}$ sodium pentobarbital and $10 \mathrm{mg} / \mathrm{kg}$ chlorprothixene; the dose was adjusted to eliminate the pedal withdrawal reflex. Additional intraperitoneal injections of $5-25 \mathrm{mg} / \mathrm{kg}$ sodium pentobarbital were administered as necessary throughout the duration of the experiment. Dilute $0.1 \%-0.5 \%$ lidocaine, not exceeding $5 \mathrm{mg} / \mathrm{kg}$, was topically applied at all points at which incisions made. Brain edema was prevented by intraperitoneal injection of $20 \mathrm{mg} / \mathrm{kg}$ dexamethasone. Animals were warmed with a thermostatically controlled heat lamp to maintain body temperature at $37^{\circ} \mathrm{C}$. A tracheotomy was performed. The head was placed in a mouse adaptor (Stoelting). A cisternal drain was performed. Soft tissue over the skull was retracted. A craniotomy and durotomy were performed to expose visual cortex. The cortical surface was kept moist with normal saline or $4 \%$ agarose in normal saline. Primary visual cortex was located and mapped by multiunit extracellular recording with a parylene-coated tungsten electrode (Micro Probe). The boundaries were identified by the characteristic gradient in the receptive field locations (Gordon and Stryker, 1996). Cat experiments were performed as previously described using anesthetized, paralyzed female cats $(2-2.5 \mathrm{~kg}$ ) (Priebe and Ferster, 2006). Anesthesia was induced with ketamine $(5-15 \mathrm{mg} / \mathrm{kg})$ and acepromazine $(0.7 \mathrm{mg} / \mathrm{kg})$, and cannulae were inserted into the saphenous veins and the trachea. After intravenous administration of sodium thiopental $(10-20 \mathrm{mg} / \mathrm{kg})$, the head was fixed in a stereotaxic frame. Two measures were taken to increase the stability of recordings: (1) the animal's thoracic vertebrae were suspended from the stereotaxic frame; and (2) a pneumothoracotomy was performed. The animal was maintained under anesthesia using an intravenous infusion of sodium thiopental $(1-2 \mathrm{mg} / \mathrm{kg} / \mathrm{h})$ for the duration of the experiment. To minimize drift in eye position, paralysis was maintained with an infusion of vecuronium bromide (Norcuron, $0.2 \mathrm{mg} / \mathrm{kg} / \mathrm{h}$ ) or gallamine $(10 \mathrm{mg} / \mathrm{kg} / \mathrm{h})$. Body temperature was kept at $38.2^{\circ} \mathrm{C}$ with a thermostatically controlled heat lamp. The nictitating membrane was retracted with the application of phenylephrine hydrochloride and the pupils were dilated using topical atropine. The corneas were protected by contact lenses with artificial pupils ( $4 \mathrm{~mm}$ diameter). Supplementary lenses were selected by direct ophthalmoscopy to focus the display screen onto the retina.

All procedures were approved by The University of Texas at Austin Institutional Animal Care and Use Committee.

Whole-cell recordings. Blind whole-cell recordings in vivo were obtained (Pei et al., 1991; Ferster and Jagadeesh, 1992; Margrie et al., 2002). As a reference electrode, a silver-silver chloride wire was inserted into muscle near the base of the skull, and covered with $4 \%$ agarose in normal saline to reduce changes in the surrounding fluid and concomitant changes in associated junction potentials. The potential of the CSF was assumed to be uniform and equal to that of the reference electrode. Pipettes (5-10 M $\Omega$ ) were pulled from $1.5 \mathrm{~mm}$ outer diameter, $1 \mathrm{~mm}$ inner diameter KG-33 borosilicate glass capillaries (King Precision Glass) on a P-97 micropipette puller (Sutter Instruments) to record from neurons $250-650 \mu \mathrm{m}$ below the cortical surface. To record membrane potential and spike responses, pipettes were filled with (in $\mathrm{mM}$ ) 135 K-gluconate, $4 \mathrm{NaCl}$, 0.5 EGTA, $2 \mathrm{MgATP}, 10$ phosphocreatine disodium, and 10 HEPES, pH adjusted to 7.3 with $\mathrm{KOH}$ (Sigma-Aldrich). For estimating synaptic excitation and inhibition, pipettes contained (in mM) 135 Cs-methanesulfonate, 4 TEA-Cl, 0.5 QX-314, 0-4 BAPTA, 2 MgATP, 10 phosphocreatine disodium, and 10 HEPES, pH 7.3 with $\mathrm{CsOH}$ (Sigma Aldrich) in mice (Tan and Wehr, 2009; Adesnik and Scanziani, 2010; Liu et al., 2010); and 130 Cs-methanesulfonate, 7 QX-314, 2
$\mathrm{MgCl}_{2}, 5 \mathrm{HEPES}, 1.1$ EGTA, $0.1 \mathrm{CaCl}_{2}$, and $4 \mathrm{Na}_{2} \mathrm{ATP}$ in cats. Current and voltage clamping were performed with a MultiClamp 700B patchclamp amplifier (Molecular Devices). Current flow out of the amplifier into the patch pipette was considered positive. In recordings with the potassium-based pipette solution, resting membrane potentials ranged from -50 to $-80 \mathrm{mV}$. In recordings with the cesium-based pipette solution, resting membrane potentials ranged from -10 to $-40 \mathrm{mV}$; when recording membrane potential responses, constant current was injected to hyperpolarize the membrane potential (in the absence of a visual stimulus) to -60 to $-80 \mathrm{mV}$. Series resistances ranged from 30 to 100 $\mathrm{M} \Omega$ for current-clamp recordings, and 30-60 $\mathrm{M} \Omega$ for voltage-clamp recordings.

Stimuli. Visual stimuli were generated by a Macintosh computer using the Psychophysics toolbox (Brainard, 1997; Pelli, 1997) for Matlab and presented on a Sony GDM f-520 video monitor placed 25 or $50 \mathrm{~cm}$ in front of the animal's eyes. The video monitor had a non-interlaced refresh rate of $120 \mathrm{~Hz}$ and a spatial resolution of $1280 \times 1024$ pixels, which subtended $38 \mathrm{~cm}$ horizontally and $30 \mathrm{~cm}$ vertically. The video monitor had a mean luminance of $25 \mathrm{~cd} / \mathrm{cm}^{2}$. Grating stimuli were presented for $1.5 \mathrm{~s}$ (mouse) or $4 \mathrm{~s}$ (cat), preceded and followed by $1.25 \mathrm{~s}$ blank (mean luminance) periods. The receptive fields of neurons were initially mapped by hand for receptive field position and size. Orientation selectivity was measured near optimal spatial frequency and spatial position, at $2 \mathrm{~Hz}$ temporal frequency, and at $90 \%$ contrast.

Analysis. Excitatory and inhibitory conductances were estimated as previously described (Priebe and Ferster, 2006; Liu et al., 2009; Tan and Wehr, 2009; Adesnik and Scanziani, 2010). In all cases, the conductances represent the change in conductance from the mean conductance during the blank period. For voltage-clamp recordings, EPSCs were measured at a holding potential of $-80 \mathrm{mV}$, near the inhibitory reversal potential. IPSCs were measured at a holding potential of $10 \mathrm{mV}$, near the excitatory reversal potential of $\sim 0 \mathrm{mV}$, as outward current with magnitude at least 2 SDs greater than the current during blank periods. For current-clamp conductance measurements, membrane potential recordings were made while injecting at least three different current levels. Error bars for these measurements were based on a bootstrap analysis. For both currentclamp and voltage-clamp estimates of conductance, a reversal potential of $0 \mathrm{mV}$ was assumed for excitatory conductances and -70 for inhibitory conductances. Varying either reversal potential assumption by $10 \mathrm{mV}$ does not change any of the conclusions made here. To compare estimates of subthreshold membrane potential from conductance to recorded membrane potential, raw records were low-pass filtered using a filter with a cutoff at $100 \mathrm{~Hz}$ to remove spikes. Spikes were identified on the basis of the large deflections in membrane potential. Error bars represent SEM unless otherwise indicated.

\section{Results}

\section{Orientation selectivity is broader for membrane potential than for spike rate}

To explore the cortical circuitry and computation responsible for the generation of orientation selectivity in mouse V1, we recorded intracellularly from single V1 neurons using whole-cell recordings in anesthetized mice in vivo. For each neuron, we presented drifting sine-wave gratings of varying orientations and measured both the subthreshold membrane potential fluctuations as well as the spiking response of the neuron. The membrane potential response represents the combination of both excitatory and inhibitory synaptic inputs to the neuron, and as such, reflects an estimate of the combined selectivity, relative to the output selectivity given by the neuron's spike rate. To compare the input and output orientation selectivities, we separated recorded responses into spike rate (extracted from the large deviations of membrane potential) and membrane potential (extracted by low-pass filtering the raw membrane potential trace), and plotted both responses as a function of stimulus orientation. We found that mouse V1 neurons displayed a wide range of orientation selectivity (Fig. $1 A-C$ ). The first example neuron (Fig. 

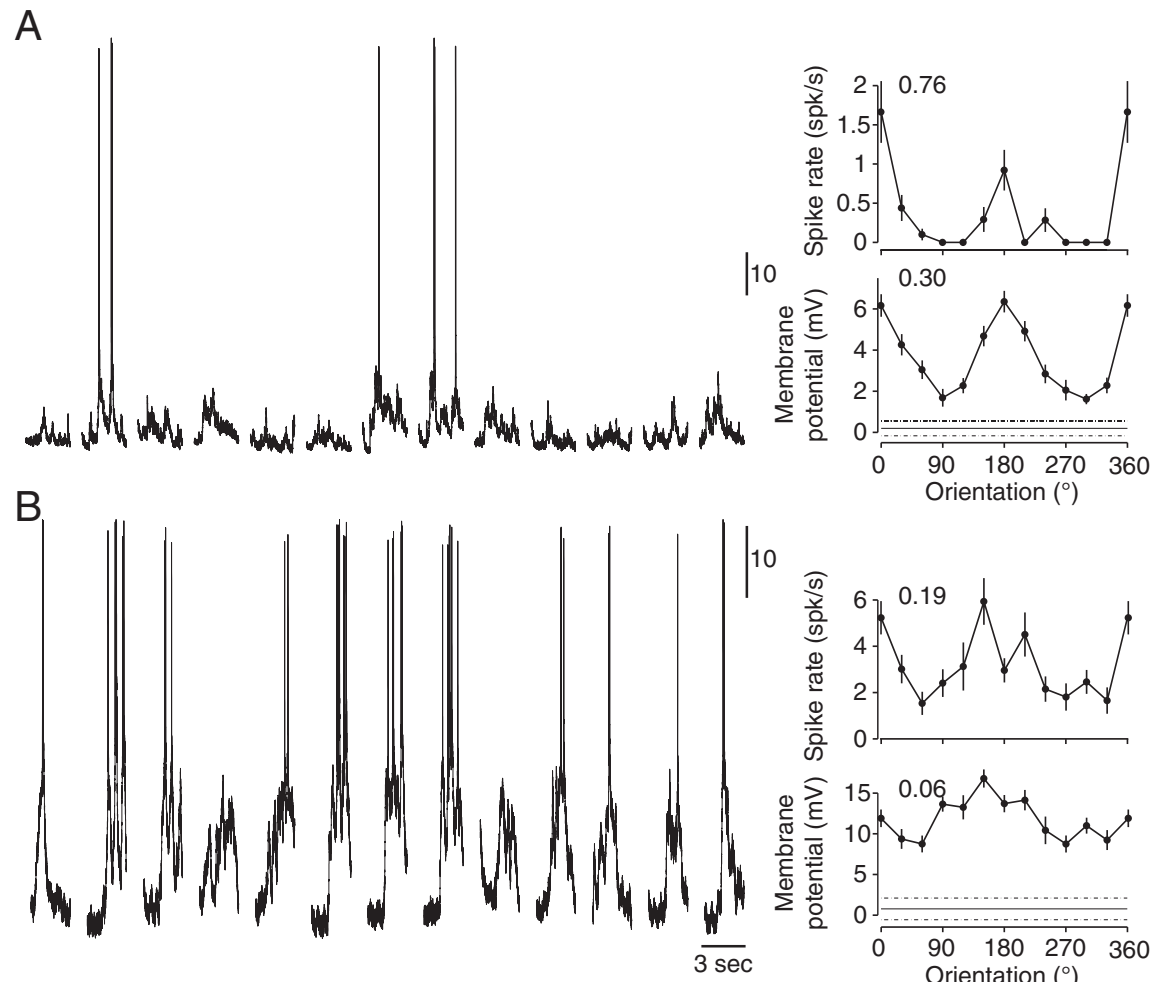

C
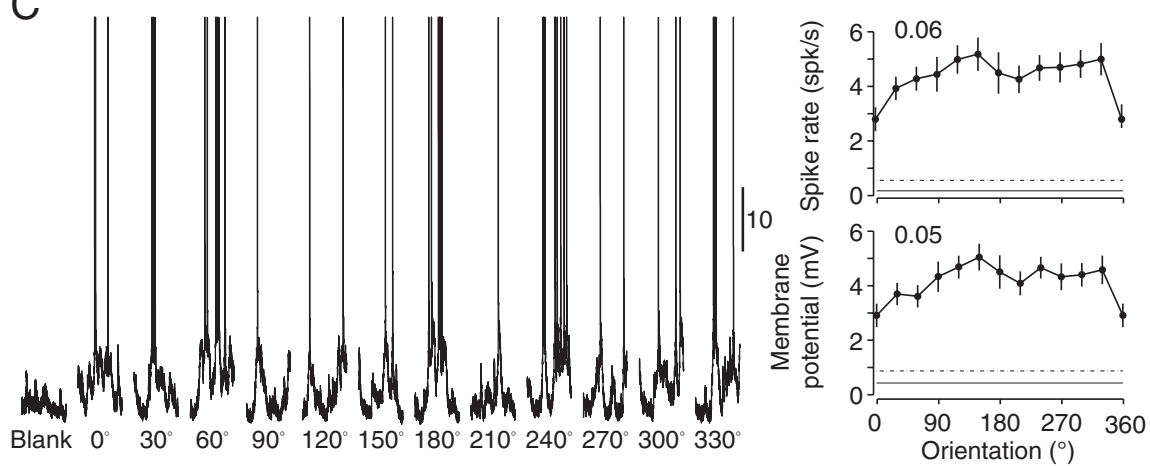

Figure 1. Membrane potential orientation tuning. $\boldsymbol{A}-\boldsymbol{C}$, Membrane potential responses for a well tuned $(\boldsymbol{A})$, moderately tuned $(\boldsymbol{B})$, and poorly tuned $(\boldsymbol{C})$ neuron. The resting membrane potentials for each of these cells was $-69.9 \mathrm{mV}(\boldsymbol{A}),-59.3 \mathrm{mV}(\boldsymbol{B})$, and $-65.6 \mathrm{mV}(\boldsymbol{C})$. For each neuron, the left panel shows membrane potential responses to gratings of different orientations; the right top and bottom panels show spike rate and membrane potential orientation tuning curves, respectively; the 0SI of each tuning curve is at the curve's top left.

1A) was highly selective for stimulus orientation, spiking only for stimulus orientations near $0^{\circ}$ and $180^{\circ}$. The underlying membrane potential depolarization was also orientation selective, with greatest depolarizations elicited by stimulus orientations near $0^{\circ}$ and $180^{\circ}$. All stimulus orientations elicited depolarizations As reflected in the individual example traces, the example neuron's spiking orientation selectivity appeared higher than its membrane potential selectivity (Fig. $1 A$, right).

To make quantitative comparisons of the degree of orientation selectivity for a neuron's membrane potential responses and its spike rate responses, we used the orientation selectivity index (OSI) for both:

$$
\text { OSI }=\frac{\sqrt{\left(\sum R(\Theta) \sin (2 \Theta)\right)^{2}+\left(\sum R(\Theta) \cos (2 \Theta)\right)^{2}}}{\sum R(\Theta)},
$$

where $\Theta$ is stimulus orientation and $R(\Theta)$ indicates the evoked peak response ( $F_{1}+$ DC, see Materials and Methods $)$ to each grating (Swindale, 1998). A neuron that responds exclusively to a single orientation will have an OSI $=1$, whereas a neuron that responds equally to all orientations will have an OSI $=0$. For example, for the highly selective V1 neuron in Figure $1 A$, its spike rate OSI is 0.76 , whereas the membrane potential OSI is 0.30 , reflecting the increased selectivity of spike rate relative to membrane potential (Carandini and Ferster, 2000; Priebe and Ferster, 2008; Jia et al., 2010).

The example neuron in Figure $1 A$ is one of the most orientation-selective neurons in our recorded sample population $(n=20)$. The majority of our recorded neurons displayed prominent membrane potential responses to all orientations (Fig. $1 B, C$ ), though biases in membrane potential fluctuations did still indicate orientation preference (Fig. $1 B$ ). For six neurons, a preference for orientation was not clear from either the membrane potential or the spike rate responses. Note that while orientation selectivity was not prominent for the subgroup with low selectivity, the lack of selectivity did not reflect a lack of visually evoked-response.

Across our sample population of recorded neurons, spike rate OSI ranged from 0.04 to 0.76 , with a median of 0.31 (Fig. 2A, gray), a value similar to estimates of selectivity based on functional imaging and extracellular single-unit recording in mouse V1 (Sohya et al., 2007; Kerlin et al., 2010; Runyan et al., 2010) in mouse V1. Also across our sample population, the orientation selectivity for membrane potential responses was much lower than for spike rate responses, with membrane potential OSI ranging from 0.02 to 0.34 , with a median of 0.09 (Fig. $2 B$, gray). Orientation selectivity, measured by OSI, was consistently lower for membrane potential than spike rate (paired $t$ test, $p<0.01$ ), on a neuron-by-neuron basis. Despite this difference in the degree of selectivity between spike rate and membrane potential, the membrane potential selectivity was highly correlated to spike rate selectivity $\left(R^{2}=0.70, p<0.01\right.$; Fig. $2 D)$. Finally, in the population of 15 neurons that evoked spiking responses, we classified 10 as simple cells by the modulation ratio $\left(F_{1} / F_{0}\right)$. The degree of orientation selectivity was not related to the modulation ratio of the neurons $\left(R^{2}=0.05, p>0.35\right)$, nor to the resting membrane potential $\left(R^{2}=0.02, p>0.5\right)$.

To compare the degree of orientation selectivity present in mouse V1 neurons with that documented in cat V1 neurons, we also obtained intracellular records from cat $\mathrm{V} 1$ in vivo using drifting gratings. To facilitate a comparison across species, the same metric, OSI, was also calculated from our recorded population of neurons $(n=31)$ in cat V1. Spiking orientation selectivity was higher in cat V1 than mouse V1 (Fig. $2 A$, cat median spiking OSI $=0.83$, mouse median spiking OSI $=0.31$, unpaired $t$ test, $p<0.01)$. Membrane potential selectivity in the cat was higher 
than membrane potential selectivity in the mouse (Fig. $2 B$, unpaired $t$ test, $p<0.01$ ). As in mouse V1, cat V1 membrane potential orientation selectivity (median $=0.28$ ) was lower than spike rate orientation selectivity. For neurons in both cat and mouse V1, orientation selectivity based on spike rate was higher than that based on membrane potential. Two trends are evident in these data: first, neurons in cat $\mathrm{V} 1$ are more orientation selective than neurons in mouse V1 when assayed via membrane potential or spike rate, and second, for both species membrane potential selectivity is broader than spike rate selectivity. These trends exist whether the neurons are compared individually or whether population average tuning curves are generated.

The discrepancy between orientation selectivity of membrane potential and of spike rate suggests that a neuron's threshold nonlinearity that separates these responses is enhancing selectivity (Gardner et al., 1999; Carandini and Ferster, 2000; Priebe and Ferster, 2008; Jia et al., 2010). To test this, we first described the relationship between mean membrane potential and spike rate across all stimulus conditions with a threshold nonlinearity:

$$
R\left(V_{\mathrm{m}}\right)=k\left[\overline{V_{\mathrm{m}}}-V_{\text {rest }}\right]_{+}^{p},
$$

where $R$ is the spike rate, $V_{\mathrm{m}}$ the membrane potential, and $V_{\text {rest }}$ the resting membrane potential; free parameters $k$, the gain factor, and $p$, the power-law exponent, were set by least-squares fitting. Neurons exhibited different power-law exponents, with $p$ ranging from $>5$ to $<2$ (Fig. $2 C$, top to bottom).

We used a simple model to examine how different power-law exponents affect orientation selectivity. Membrane potential orientation selectivity was modeled as a fixed width ( $\sigma=25$ degrees) Gaussian above a fixed baseline. The Gaussian's amplitude was varied to create different membrane potential OSIs. We transformed the model membrane potential responses by applying different exponents, $p=2,3$, and 5. Each of these different exponents creates a different predicted relationship between membrane potential OSI and spike rate OSI (Fig. $2 D$, blue, red, and black traces), with the higher exponent causing a steeper relationship between membrane potential OSI and spike rate OSI. Two effects that are evident in this simple threshold model: first, spike rate OSI should be higher than membrane potential OSI, and second, this relationship is very steep for when there is only mild membrane potential orientation selectivity, and it saturates for higher membrane potential selectivity. Both of these effects are present when we plot the measured membrane potential and spike rate OSI for each of our recorded neurons (Fig. 2D, mouse neurons, circles, and cat neurons, squares). Further those neurons with higher exponents (Fig. $2 D$, black symbols) tended to have a steeper relationship between OSI of membrane potential and of spike rate, than did those neurons with lower exponents (Fig. 2D, blue symbols).

Since membrane potential orientation tuning curves may differ from that assumed in the simple model, we used each neuron's membrane potential response and fit threshold nonlinearity to predict its spike OSI (Fig. $2 E$ ). The predicted values were highly correlated with the measured values $\left(R^{2}=0.77\right.$, mouse, $R^{2}=$ 0.71 , cat). Across species, the slope of the correlation (0.95) was not significantly different from $1(p>0.35)$; the $y$-intercept (0.09) was different from $0(p<0.05)$. These results suggest that the threshold nonlinearity plays a role for the increase in orientation selectivity from membrane potential to spike rate and that using a threshold applied to all stimulus conditions equally can recapitulate this increase. Despite the difference in overall orientation selectivity between neurons of cat V1 and mouse V1, it appears that the input selectivity across these neuronal populations forms a continuum, where the same threshold rule applies.

Spike threshold can be stimulus-dependent (Azouz and Gray, 2000; Wilent and Contreras, 2005b; Yu et al., 2008). Accordingly, we examined whether we could detect this for orientation tuning in the mouse. Each spike's threshold was estimated as the membrane potential at the time of maximal membrane potential acceleration before the spike (Wilent and Contreras, 2005b). The estimated spike threshold was not significantly different across orientation in any neuron (ANOVA, $p>0.07$ ).

\section{Synaptic excitation and inhibition and membrane potential have similar orientation selectivity}

While the spike rate orientation selectivity is narrower than and is predicted by the underlying membrane potential selectivity, it has been unclear how excitatory and inhibitory inputs combine at a single neuron to generate that neuron's membrane potential selectivity. To determine this synaptic basis for membrane potential orientation selectivity, and thereby spike rate selectivity, we 

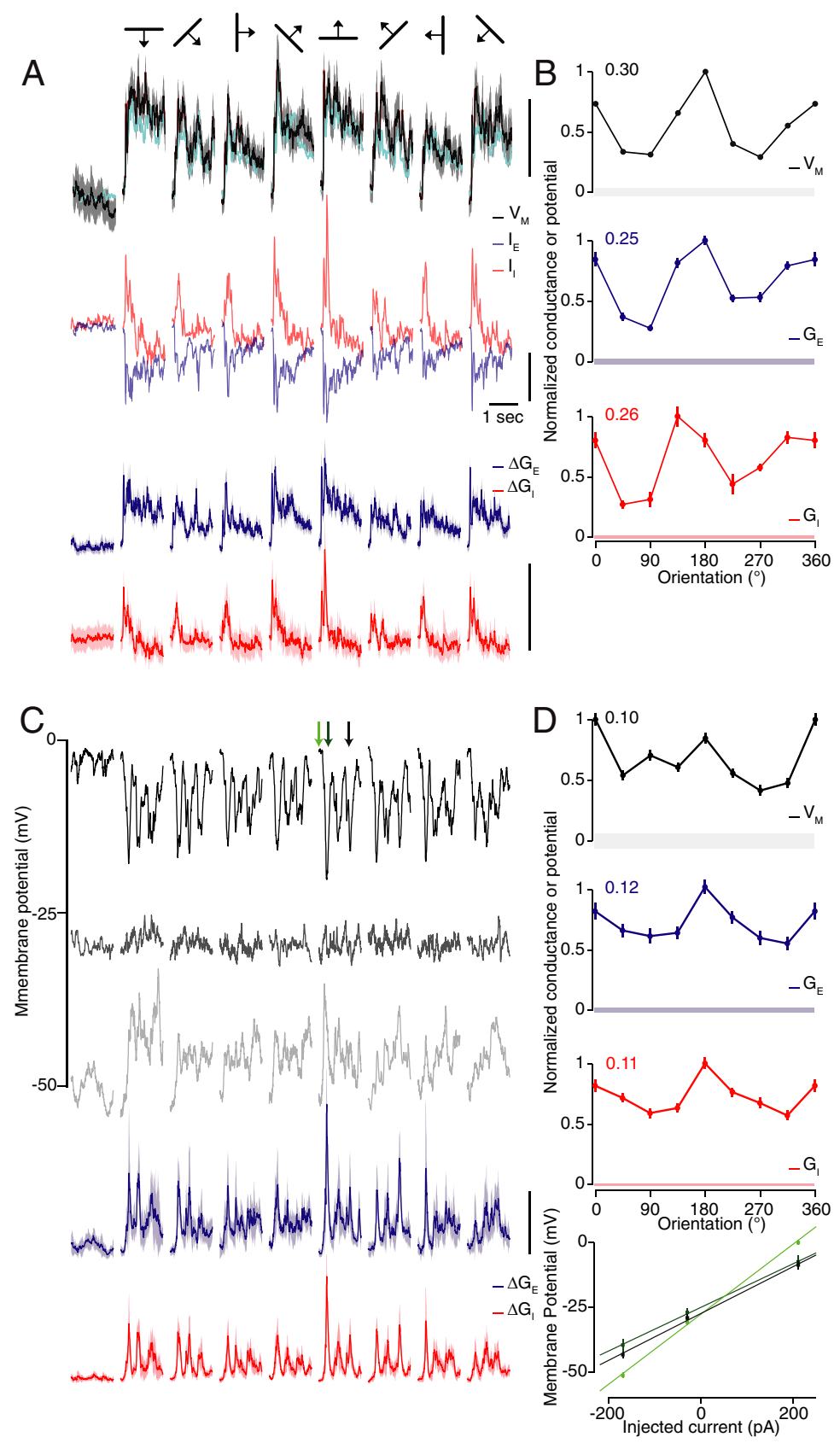

Figure 3. Excitatory, inhibitory, and membrane potential orientation selectivity. $A$, An example neuron for which conductances were estimated using voltage clamp. The top row indicates the membrane potential orientation selectivity recorded in current clamp (black traces, calibration: $2 \mathrm{mV}$ ). Predictions of membrane potential responses based on estimated conductances are also shown (cyan traces). The second row shows the currents measured when the neuron was voltage clamped at $-16 \mathrm{mV}$ (light blue) and $-81 \mathrm{mV}$ (light red, calibration: $20 \mathrm{pA}$ ). Excitatory and inhibitory conductances (blue and red traces, respectively, calibration: $0.4 \mathrm{nS}$ ) are shown for each orientation. $\boldsymbol{B}$, Orientation selectivity for membrane potential (top, peak $=1.3 \mathrm{mV}$ ), excitatory conductance (middle, peak $=0.18 \mathrm{nS}$ ), and inhibitory conductance (bottom, peak $=0.32 \mathrm{nS}$ ). Error bars indicate the SEM. $C$, An example cell for which conductances were estimated using current clamp. The top panels show the membrane potential responses as theneuron was injected with $210,-30$, or $-173 \mathrm{pA}$ (from black traces to gray traces). Excitatory and inhibitory conductances derived from those measurements are shown in the bottom traces (Ge, blue traces; Gi, red traces; calibration: $5 \mathrm{nS}$ ). Shaded regions indicate the 95\% confidence intervals derived from bootstrap analysis. $\mathbf{D}$, Orientation selectivity for membrane potential (extracted from the data taken when the neuron was hyperpolarized, peak $=12 \mathrm{mV}$ ), excitatory conductances (middle, peak $=3 \mathrm{nS}$ ), and inhibitory conductances as in $\boldsymbol{B}$ (peak $=2.2 \mathrm{nS}$ ). The inset values in these panels indicate the OSI for each tuning curve. The relationship between injected current and observed membrane potential is plotted for the three epochs indicated by arrows in $\boldsymbol{C}$.

performed whole-cell recordings in vivo in mouse $\mathrm{V} 1$ with intrinsic channel blockers to isolate the excitatory and inhibitory inputs (see Materials and Methods). These recordings were performed either in voltage clamp (Fig. $3 A$ ) or in current clamp (Fig. 3B), but the strategy was the same in both recording conditions; the excitatory and inhibitory synaptic conductances can be inferred by measuring the visually evoked responses while the neuron is at varying distances from the reversal potentials of excitation and of inhibition (Hirsch et al., 1998; Anderson et al., 2000; Monier et al., 2003; Haider et al., 2006; Priebe and Ferster, 2006). For example, responses recorded while the neuron is held at a membrane potential near the excitatory reversal potential are dominated by inhibitory input, since the driving force for those inputs is large relative to excitation. Conversely the responses measured while the neuron is held at a membrane potential near the inhibitory reversal potential are dominated by excitatory input, since the driving force for those inputs is now large relative to inhibition.

Orientation selectivity was evident from currents recorded when a neuron was voltage clamped at a depolarized voltage (Fig. $3 A$, light red) or at a hyperpolarized voltage (Fig. $3 A$, light blue). Using these current records we estimated excitatory and inhibitory conductances (Fig. $3 A$, blue and red traces) for each stimulus orientation. Excitatory and inhibitory inputs both displayed orientation selectivity, and appeared to have maximal responses at similar stimulus orientations (Fig. 3A). It is important to note that these measures of inhibitory and excitatory input represent the aggregate, convergent input from many presynaptic inhibitory and excitatory neurons, and not individual presynaptic neurons. To compare the orientation selectivity of excitatory and inhibitory inputs with that of the resultant membrane potential, we also recorded the membrane potential by switching the recording into current-clamp mode; as cesium in the intracellular solution blocks intrinsic potassium channels and raises the resting membrane potential, we injected current into the neuron to restore a resting membrane potential of approximately $-70 \mathrm{mV}$ (Fig. 3A, black traces). Our membrane potential records revealed visually evoked fluctuations with a similar orientation selectivity as we observed in both the excitatory and inhibitory inputs to the same neuron.

To assess the validity of the linear model used to estimate conductances, we used the linear model based on the estimated conductances to predict the membrane potential. The predicted membrane potential (Fig. $3 A$, top row, cyan traces) overlapped the actual membrane potential obtained by switching to current-clamp recording mode (Fig. $3 A$, top row, black traces), accounting for $96 \%$ of its variance. We obtained membrane potential records in 


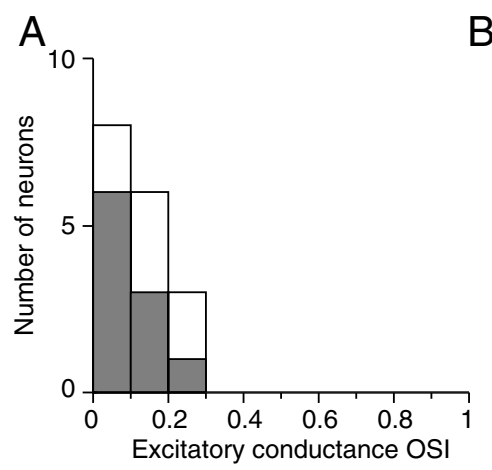

$\mathrm{E}$
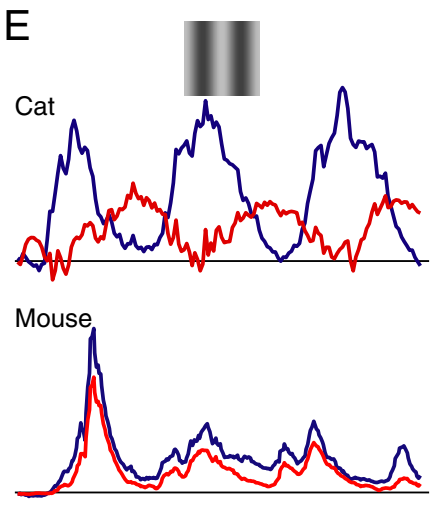

B

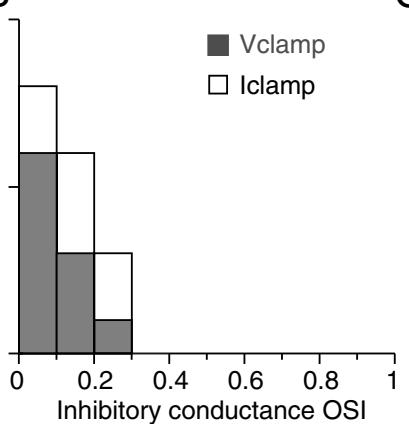

C

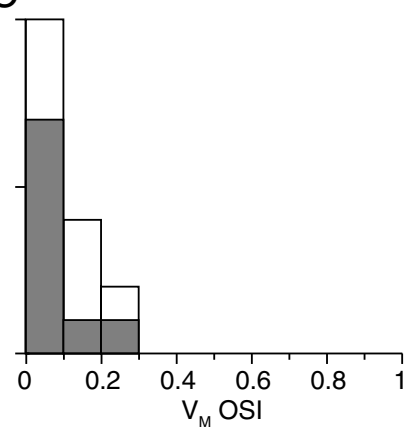

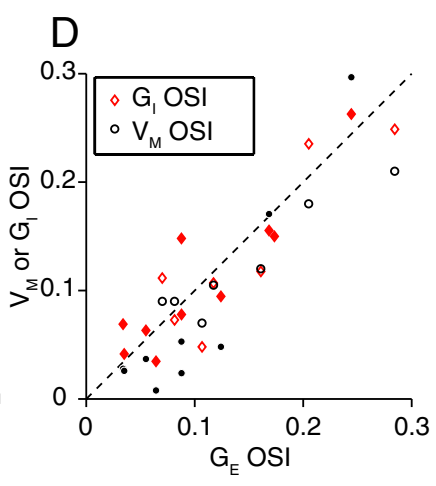

$F$ os

Excitation

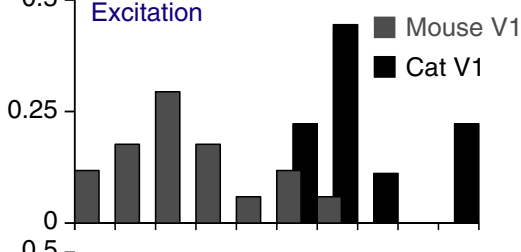

$-\Delta G_{E}$

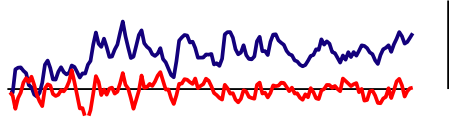

$-\Delta G_{1}$
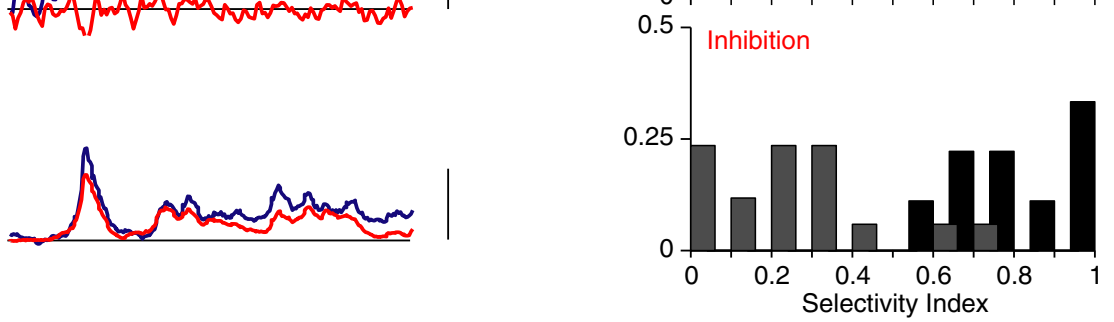

Figure 4. Excitatory, inhibitory, and membrane potential orientation tuning distribution. A, Histogram of Ge OSI. B, Histogram of Gi OSI. C, Histogram of membrane potential OSI. D, Gi OSI (red diamonds) and membrane potential OSI (black circles) versus Ge OSI. Open symbols indicate measurements from current-clamp recordings, filled symbols indicate measurements from voltageclamp recordings. $\boldsymbol{E}$, Conductance measurements for excitation (blue traces) and inhibition (red traces) for the preferred orientation (left column) and the orthogonal orientation (right column). The first row shows an example cat neuron, the second row shows an example mouse neuron. The stimulus began at beginning of the trace and was on for the duration of the trace (1.5 $\mathrm{s}$ are shown). Calibration bars indicate the size of the conductances (cat, $2 \mathrm{nS}$, mouse, $5 \mathrm{nS}$ ). $\boldsymbol{F}, 0 \mathrm{SI}$ distribution for excitatory conductance (top panel) and inhibitory conductance (bottom panel).

7 of the 11 neurons in which conductances were estimated via voltage clamp. Across these 7 neurons, the predicted membrane potential accounted for $73 \%$ of the variance of the actual membrane potential; the predicted membrane potential lay $93 \%$ of the time within the $95 \%$ confidence limits of the actual membrane potential; OSIs of predicted and actual membrane potentials were correlated $\left(R^{2}=0.86\right)$. These suggest that the linear model provides a reasonable approximate description for this dataset.

We measured the orientation tuning of excitation, inhibition and membrane potential during a time window in which each neuron exhibited significant responses. The time window was less than the stimulus duration because inhibitory responses were often significantly different from baseline only when assessed over a shorter time window (we further describe response time courses in later sections) and was the same for all orientations The orientation tuning curves confirmed that excitation, inhibition, and membrane potential had similar orientation tuning, peaking at similar orientations and having similar tuning widths and OSIs (Fig. 3B).

Because it has been suggested that synaptic conductances estimated by voltage and current clamp may differ (Alitto and Dan, 2010), we also estimated excitatory and inhibitory conductances using the current-clamp configuration instead of voltage clamp. In the current-clamp configuration, a steady current was injected into the neuron to either depolarize or hyperpolarize the neuron during visual stimulation (Fig. 3C). Visually evoked membrane potential responses are quite distinct depending on the current injected, leading to large hyperpolarizations when the neuron is depolarized (Fig. 3C, black traces) and to large depolarizations when the neuron is hyperpolarized (Fig. 3C, gray traces). Critically, at intermediate membrane potentials, the visually evoked responses were dramatically diminished, suggesting that the excitatory and inhibitory currents occurred simultaneously and thus negated their effects on membrane potential. As in the voltage-clamped example neuron (Fig. $3 A, B$ ), orientation selectivity was evident at the level of membrane potential responses and in the excitatory and inhibitory conductances extracted from these measurements. Membrane potential, excitatory conductance, and inhibitory conductance all had very similar degrees of orientation selectivity, with the same preferences for orientation (Fig. 3D, top panels).

To generate these conductance estimates based on currentclamp recordings, a linear relationship between amount current and the observed membrane potential responses was required. This linear relationship exists in our recordings (Fig. 3D), as shown for three different response latencies (indicated by arrows in Fig. 3B), corresponding to the period before the evoked response (light green), the peak response (green), or later during the stimulus (black). For all orientations and time periods, regression accounted for $>98 \%$ of the variance in the relationship between injected current and mean membrane potential, and accounted for $>94 \%$ of the relationship between injected current and the recorded trial-by-trial membrane potential.

Across our sample of 17 mouse V1 neurons for which estimates of excitatory and inhibitory input selectivity were made, we found similar distributions of excitatory input selectivity (Fig. $4 A$; mean OSI: 0.13), inhibitory input selectivity (Fig. $4 B$; mean OSI: 0.11), and membrane potential selectivity (Fig. 4C). Of im- 
A

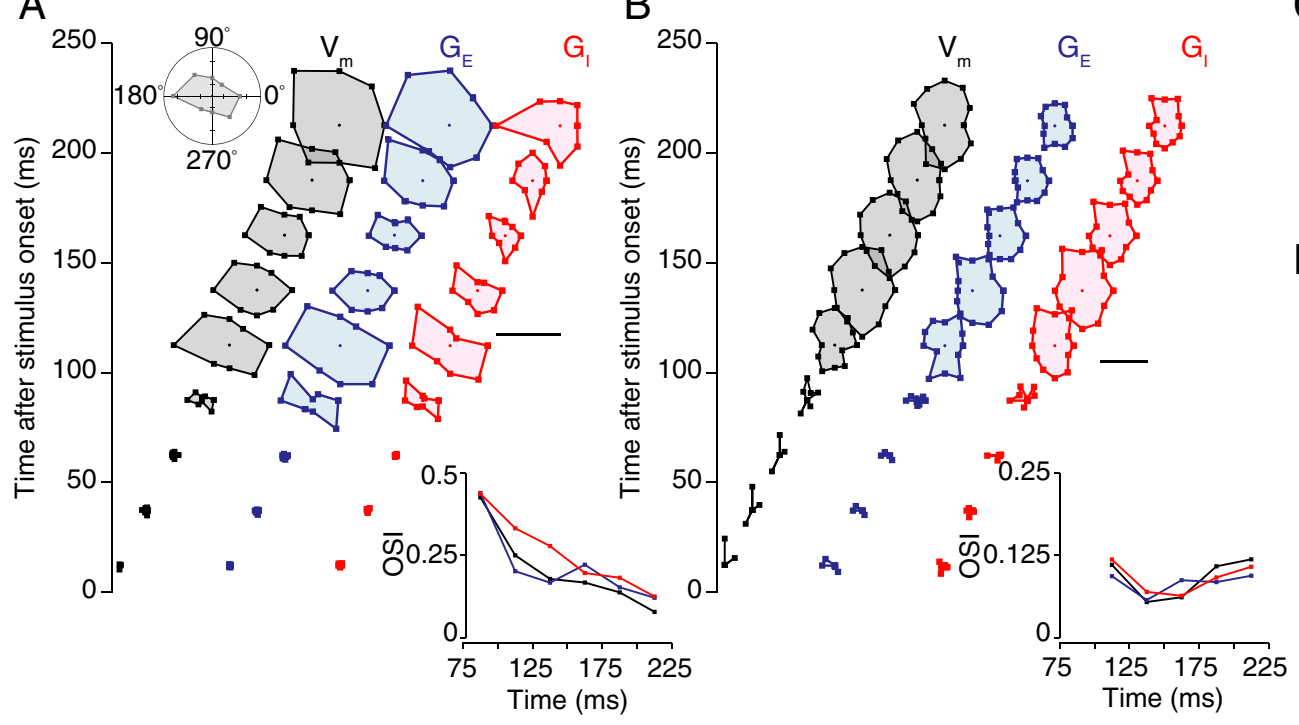

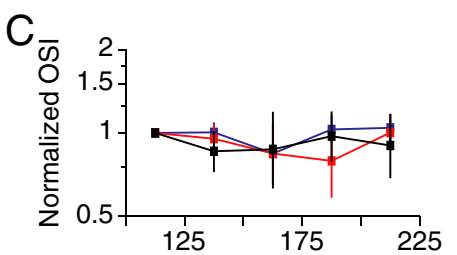
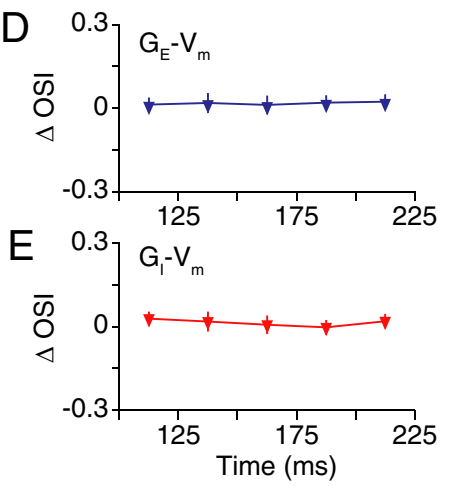

Figure 5. The time course of orientation selectivity. $A, B$, Orientation tuning is plotted for two example neurons as a function of time. Membrane potential (black), Ge (blue), and Gi (red) tunings are plotted in $25 \mathrm{~ms}$ intervals. Insets plot the 0 SI for each response type as a function of time. $\boldsymbol{A}$, Calibration: $3.5 \mathrm{mV}, 0.45 \mathrm{nS}$ (Ge) and $0.9 \mathrm{nS}$ (GI). $\boldsymbol{B}$, Calibration: $10.5 \mathrm{mV}, 2.25 \mathrm{nS}$ (Ge) and $4.5 \mathrm{nS}$ (Gi). C, Geometric mean of OSI, as fraction of initial OSI for $V_{\mathrm{m}}$ (black, $n=15$ neurons), Ge (blue, $n=17$ ), and Gi (red, $\left.n=17\right)$. D, Difference between Ge 0SI and $V_{\mathrm{m}} 0$ OSI $(n=15)$. $\boldsymbol{E}$, Difference between Gi OSI and $V_{\mathrm{m}}$ OSI $(n=15)$.

portance, however, is that the degree of selectivity for excitatory and inhibitory inputs was related such that neurons receiving very selective excitatory inputs also received very selective inhibitory inputs (Fig. $4 D$ ). Overall, excitatory and inhibitory input selectivity was highly correlated $(r=0.83)$ and the regression fit did not have a slope significantly different from 1 . These results indicate that excitation and inhibition share orientation selectivity and that inhibition is not more broadly selective than excitation.

Generating these conductances measurements required some assumptions that are critical. First, we assumed that neurons are isopotential, whereas membrane potential fluctuates throughout the dendritic arbor. The high correlation between our predicted and actual membrane potentials suggests, however, that a systematic error has not made by assuming a single compartment model (Fig. 3A). On the other hand, since Thevenin's and Norton's theorems state that single- and multi-compartment models cannot be distinguished for steady input, uncovering errors may require higher temporal frequencies than those examined here (Johnston and Wu, 1995). Second, errors in the estimated equivalent circuit parameters, such as series resistance, junction potential, and reversal potentials, will also affect estimated synaptic conductances. To reduce the impact of such errors, we have restricted our conclusions here to aspects of the conductances that are not qualitatively affected by $\pm 10 \mathrm{mV}$ changes in any reversal potential.

Comparison of orientation selectivity in V1 of mouse and cat Previous estimates of the orientation selectivity for excitation and inhibition in cat $\mathrm{V} 1$ have indicated that excitation and inhibition share orientation selectivity (Anderson et al., 2000; Monier et al., 2003), similar to what we have now shown for mouse V1. Our data do show a difference between neurons of cat V1 and mouse $\mathrm{V} 1$ when examining the degree of orientation selectivity: mouse $\mathrm{V} 1$ neurons are not as selective as cat $\mathrm{V} 1$ neurons when comparing membrane potential or spiking responses (Fig. $2 B$ ). To determine whether this is due to a difference in the selectivity of the synaptic inputs between cat and mouse, we performed conductance measurements from cat visual cortex, estimating excitatory and inhibitory conductances in simple cells in response to the preferred and null (orthogonal) stimulus orientations (Fig. 4E). Orientation tuning was probed only at preferred and null orientations in the cat. Accordingly, to compare the degree of orientation selectivity observed in mouse and cat, we computed the orientation index (OI) based on the preferred and null orientations:

$$
\mathrm{OI}=1-\frac{N}{P},
$$

where $P$ is the conductance evoked by the preferred orientation and $N$ is the conductance evoked by the null (orthogonal) orientation. If the conductance changes evoked by the preferred orientation are similar to the orthogonal orientation, the OI will be near 0 . If there is only a conductance change at the preferred orientation and not at the orthogonal orientation, the OI will be 1 . We found that in both mouse and cat excitatory and inhibitory conductances were selective (Fig. $4 F$ ), but the degree of selectivity differed between species. The OIs for cat neurons $(\mathrm{Ge}=0.71 \pm$ $0.05, \mathrm{Gi}=0.77 \pm 0.05$ ) were much higher than for mouse neurons $(\mathrm{Ge}=0.34 \pm 0.04, \mathrm{Gi}=0.27 \pm 0.05)$. These differences between cat and mouse OIs for both Ge and Gi were significant ( $p$ $<0.05$, unpaired $t$ test). These data suggest that the reduced membrane potential orientation selectivity in mouse V1 relative to cat V1 (Fig. 2) is rooted in reduced synaptic input orientation selectivity.

\section{Dynamics of synaptic excitation and inhibition}

Our analysis thus far has revealed shared orientation selectivity for excitatory and inhibitory inputs over long time periods in mouse V1, but our analysis could obscure the development of selectivity at the onset of the visual response. To investigate how orientation selectivity emerges in time, we measured orientation selectivity for membrane potential, excitatory and inhibitory conductances in $25 \mathrm{~ms}$ intervals starting with the onset of the visual stimulus (Fig. $5 A, B$ ). Initially the response amplitude for 
each measure is small, since the measurements are made before visual latency of the neurons. At $100 \mathrm{~ms}$, responses are evident for membrane potential (Fig. $5 A, B$, $V_{\mathrm{m}}$, black), and demonstrate early orientation selectivity. That orientation selectivity remains for the first $100 \mathrm{~ms}$ of response (Gillespie et al., 2001) and is also observed in both the excitatory and inhibitory synaptic inputs (Fig. $5 A, B$, blue and red). Importantly, measures of orientation selectivity, in terms of OSI, do not vary substantially between $V_{\mathrm{m}}$, excitation, and inhibition (Fig. $5 A, B$, insets). The first example neuron (Fig. $5 A$ ) does indeed have a change in orientation selectivity in time, starting at a very high selectivity and becoming less selective over the first $100 \mathrm{~ms}$, but all three measures change in concert. The second example neuron (Fig. 5B) is not highly orientation selective, but it still reveals a bias in selectivity that is shared across $V_{\mathrm{m}}$, excitation, and inhibition. Across neurons, OSI, as a fraction of initial OSI, remained constant over time for $V_{\mathrm{m}}$, excitation, and inhibition (ANOVA, $p>0.05$; Fig. $5 C)$. The difference between the OSIs of excitation and $V_{\mathrm{m}}$ remained constant over time (ANOVA, $p>0.05$; Fig. 5D), as did the difference between the OSIs of inhibition and $V_{\mathrm{m}}$ (ANOVA, $p>0.05$; Fig. $5 E$ ). Excitation and inhibition therefore share orientation selectivity in mouse cortical neurons, even at the onset of response, and any alteration of orientation selectivity over time occurs for both excitatory and inhibitory inputs.

The separation of excitatory and inhibitory conductances revealed a strong adaptation component in the synaptic inputs, which was not always evident in the membrane potential in mouse V1 neurons (see Fig. $3 A, B$ ). It is apparent that both excitatory and inhibitory currents adapt rapidly over the brief $1.5 \mathrm{~s}$ of the visual stimulus. To examine the evolution of synaptic excitation and inhibition more closely, we aligned the excitatory and inhibitory conductances evoked by the stimulus orientation that generated the largest excitatory conductance change (Fig. 6A, B). For most recorded neurons in mouse V1, we observed that inhibition was more strongly reduced over the time course of stimulus presentation than was excitation (Fig. 6A,B).

To characterize the degree of this adaptation, we compared the peak excitatory and inhibitory currents during two $500 \mathrm{~ms}$ windows (corresponding to a single stimulus period). The first (early) window occurred right at the response onset and the second (late) window was the final $500 \mathrm{~ms}$ of visual stimulation (indicated by the shaded regions in Fig. 6A,B). Across our sample of neurons in mouse $\mathrm{V} 1$, the excitatory conductances were larger during the early window than during the late window (Fig. $6 C$, Wilcoxon signed rank test, $p<0.05$ ), as were the inhibitory conductances (Fig. 6D, Wilcoxon signed rank test, $p<0.05$ ). Since it appears that inhibition adapts to a greater degree than excitation, we measured the ratios of late-to-early responses for excitation and inhibition (Fig. 6E). For $30 \%$ of our recorded mouse V1 neurons, both excitation and inhibition adapted by equal amounts, but for most neurons, inhibition was reduced to a greater extent than excitation. In only one recorded neuron did inhibition adapt substantially less than excitation. Across our sample population, adaptation reduced inhibitory conductance more than excitatory conductance (Wilcoxon signed rank test, $p<0.05)$.

The degree of excitatory and inhibitory adaptation we found in mouse V1 neurons stands in sharp contrast to the degree of adaptation observed in cat V1 neurons (Fig. $4 E$ ), which exhibited little to no adaptation either for inhibition or excitation. We performed the same analysis comparing the peak conductances for the first response cycle to the third response cycle and found that the excitatory conductance was reduced on average by $4 \%$ and the inhibitory conductance was reduced on average by $11 \%$. These mild reductions in excitatory and inhibitory conductances of cat V1 neurons were not statistically significant ( $p>0.15$, paired $t$ test, $n=14$ ).

Drifting gratings at the preferred orientation elicited strong modulations some neurons in mouse $\mathrm{V} 1$ at the temporal frequency of the grating ( $2 \mathrm{~Hz}$, Fig. $3 \mathrm{C}$ ). Such modulations are also observed in simple cells of cat V1, where it is thought that excitation and inhibition interact asynchronously to generate a modulation. In cat simple cells, one phase of the drifting grating elicits an increase in excitation, while the opposite phase is marked by a decrease in excitation and an increase in inhibition (Fig. 7A). The degree that excitation and inhibition are out of phase is illustrated by measuring the phase of the modulation at $2 \mathrm{~Hz}$, examined for the first $1.5 \mathrm{~s}$ of the visual stimulus (Fig. $7 A$, bottom). If excitation and inhibition were completely out of phase, the difference in phase angle would be $180^{\circ}$ and the arrows in the phase plot would be pointed in opposite directions. For the example cat simple cell in Figure $7 A$, the phase difference is 143 degrees, but it is clearly the case that the timing of excitation and inhibition is quite distinct (Fig. 7A, red and blue traces). The time course of the interaction between excitatory and inhibitory input was different in mouse V1. For the example neuron shown in Figure $7 B$, excitation and inhibition modulate synchronously instead of asynchronously. That is, the phase of the excitatory and inhibitory inputs are similar, having difference of only $3^{\circ}$. This difference in dynamics can be seen easily when examining the membrane potential responses when the neuron is moved to an intermediate membrane potential (Fig. $7 A, B$, gray traces). For the cat, the stimulus still evokes a large membrane potential modulation be- 


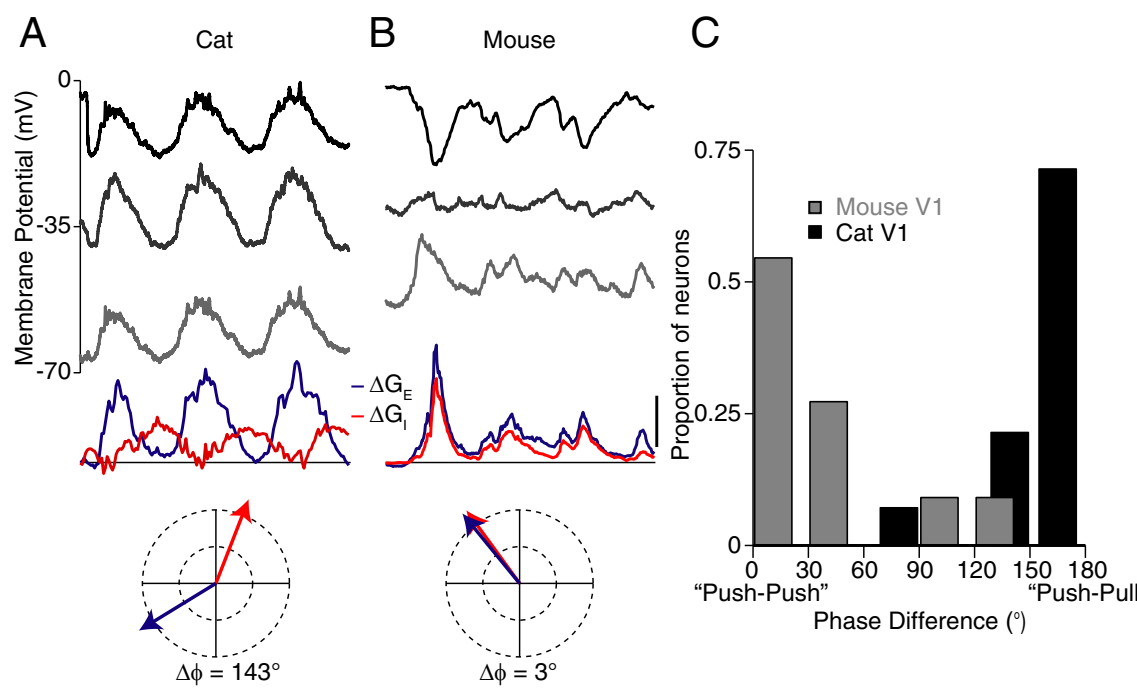

Figure 7. The timing of excitatory and inhibitory conductances. $A$, The top traces show the membrane potential responses of a cat simple cell to its preferred orientation, recorded while injecting different amounts of current $(180,0$, and $-180 \mathrm{pA})$ to extract excitatory and inhibitory conductances. The drifting grating started at the beginning of the trace with a temporal frequency of $2 \mathrm{~Hz}$. The period of stimulation shown is $1.5 \mathrm{~s}$. Excitatory (blue) and inhibitory (red) conductances based on those membrane potential responses show asynchronous excitation and inhibition. The phase angles for excitation (blue) and inhibition (red) are shown as vectors on a normalized polar plot (bottom panel). $\boldsymbol{B}$, As in $\boldsymbol{A}$, but for an example mouse neuron (currents: $-173,-30,210 \mathrm{pA}$ ). The calibration bar indicates the size of the conductances (cat, $2 \mathrm{nS}$, mouse, $5 \mathrm{nS})$. The neuron in $\boldsymbol{B}$ is the same as in Figure 3C. C, A histogram of the difference in phase angle for excitation and inhibition for mouse neurons (gray) and cat neurons (black, $n=14$ ).

cause the excitatory and inhibitory inputs are asynchronous. In contrast, for the mouse the excitatory and inhibitory inputs are synchronous, and thus at intermediate membrane potentials, there is little modulation in the evoked response. The excitatory and inhibitory inputs combine and cancel their effects on membrane potential.

We examined the difference in phase for those neurons in mouse V1 that had membrane potential modulation amplitudes $>2 \mathrm{mV}$ (11/17). (Priebe et al., 2004). Across our sample population, the relative phase of excitation and inhibition varied but the mouse neurons were characterized by relatively synchronous excitatory and inhibitory modulations (mean phase $=39 \pm 12.6^{\circ}$, $n=11$ ), while cat simple cells were characterized by out-of-phase modulations (mean phase difference $=157 \pm 7^{\circ}, n=14$, significantly different from the mouse, unpaired $t$ test, $p<0.01$ ). Thus, the temporal interplay between excitation and inhibition in response to drifting gratings is distinct between cat and mouse V1 neurons.

\section{Discussion}

The emergence of orientation selectivity in mammalian V1 has been studied to understand how synaptic organization gives rise to cortical computations. Neurons in mouse V1, as in other mammals, are selective for stimulus orientation, although we have demonstrated that mouse V1 orientation selectivity is weaker than that of cat V1 (Fig. 2), whether the comparison is made for membrane potential selectivity or for spike rate selectivity. Nevertheless, in both cat and mouse V1, a clear distinction exists between orientation selectivity at the level of membrane potential and spike rate: spike rate selectivity is always higher when compared to membrane potential selectivity. This increase in orientation selectivity is precisely what is expected given the presence of a simple threshold nonlinearity in the cortical neuron (Fig. 2C-E) (Carandini and Ferster, 2000; Priebe and Ferster, 2008; Jia et al., 2010). An orientation-independent spike thresh- old captured essential aspects of the enhanced orientation selectivity of the spike response over that of the membrane potential. This may be due both to the relatively long time windows used to assess orientation selectivity and to the variability of the membrane potential response in those windows. In contrast, stimulusdependent spike thresholds are thought to be most important when synaptic input is highly synchronous (Azouz and Gray, 1999; Wilent and Contreras, 2005a; Yu et al., 2008).

Our measures of mouse and cat orientation selectivity match those from previous reports. In particular, our mouse spike OSI distribution is consistent with recent studies using calcium imaging (Sohya et al., 2007; Kerlin et al., 2010) (Wilcoxon rank-sum, $p>0.05)$. Our intracellular records are from layer II/III and IV (mean subpial depth $342 \pm 120 \mu \mathrm{m}$ ), while the imaging records are from layer II/III, which has a degree of orientation selectivity similar to that of layer IV (Niell and Stryker, 2008). Our cat measurements of OSI also match previous reports. The degree of spike orientation tuning width, based on half-width at halfheight, did not differ from the distributions reported by Gardner et al. (1999) or by Carandini and Ferster (2000) (Wilcoxon rank-sum, $p>0.05$ ) (Gardner et al., 1999; Carandini and Ferster, 2000). The match of our records to previous cat and mouse reports suggests that the input selectivities we observe underlie a significant proportion of the output selectivities observed in other studies. We note, however, that the mean spike rate OSI of our sample was approximately half that observed in mouse layers II/III and IV using extracellular microelectrodes (Niell and Stryker, 2008), which may indicate errors in our estimates of laminar position, as OSI is lower in layer $\mathrm{V}$ than in other layers.

Uncovering the synaptic basis of the orientation selectivity represented by the inhibitory and excitatory inputs to neurons of mouse V1 provides critical constraints on models of orientation selectivity in V1. We found that excitation, inhibition, and membrane potential were similarly selective within a cortical neuron, even at the shortest response intervals. Because the degree of excitatory input selectivity derived in our measures of aggregate excitatory input was weaker than the selectivity of the spike rate of individual V1 excitatory neurons, our data suggest that there is a convergence of excitatory inputs with distinct orientation preferences (see also Jia et al., 2010). The degree of inhibitory selectivity is also consistent with convergence from input neurons with different preferences for orientation, but it is still incompletely known how selective the inhibitory neurons are in mouse V1 (Sohya et al., 2007; Kerlin et al., 2010; Runyan et al., 2010; Zariwala et al., 2011). Imaging studies suggest that the spiking responses of mouse V1 inhibitory neurons are less selective than excitatory neurons, with OSI values only slightly higher (OSI = 0.13 ) than our estimates of inhibitory selectivity (OSI $=0.09)$. As with the excitatory input, it is important to emphasize that we are measuring the selectivity of the aggregate inhibitory synaptic input to the recorded neuron. The selectivity of that aggregate input is determined primarily by two factors: (1) the diversity in orientation preference of the individual presynaptic inputs and (2) the 
tuning width of those presynaptic inputs. Similar selectivity for excitation and inhibition can emerge, even if the constituent inhibitory and excitatory inputs have different degrees of selectivity. If inhibitory neurons have broader selectivity than excitatory neurons (Sohya et al., 2007; Kerlin et al., 2010), our results suggest that the diversity of orientation preference among the inhibitory neurons should be less than the excitatory neurons (Fig. $8 A$ ). If, on the other hand, inhibitory neurons have similar degrees of orientation selectivity as excitatory neurons (Runyan et al., 2010), our results suggest a similar diversity of orientation preference among excitatory and inhibitory inputs (Fig. 8 B). From our measurements we do rule out the lateral inhibition model that relies on the convergence of inhibitory and excitatory inputs with distinct orientation preferences (Fig. 8C). In none of our recorded neurons did we observe differences in orientation selectivity consistent with a lateral inhibition model. Inhibition's shared selectivity with excitation does not exclude inhibition as a major component in the emergence of orientation selectivity. Indeed our measurements of shared selectivity suggest inhibition may be essential for controlling response gain and suppressing responses to the nonpreferred orientations (Carandini et al., 1997, 1998; Monier et al., 2003).

Our observations of the inhibitory and excitatory input dynamics suggest a complex interplay of these components over the duration of the visual stimulus. Neurons in mouse V1 are characterized by transient excitatory, inhibitory, and membrane potential responses. We observed that the inhibitory inputs to a neuron tended to adapt at a faster rate than the neuron's excitatory inputs, but both underwent profound reductions in amplitude over time, despite the continued presence of the visual stimulus. The differential dynamics between excitation and inhibition may reflect a role for inhibition in stabilizing the network as excitatory afferent input is increased with presentation of the visual stimulus (Tsodyks et al., 1997; Ozeki et al., 2009; Haider et al., 2010). While it is still unclear how much of the overall adaptation is a direct result of the afferent inputs from the thalamus, if excitatory and inhibitory cortical neurons receive the same afferent input the discrepancy in adaptation must be a result of differences in the excitatory and inhibitory cortical populations.

An essential feature of simple cells in cat V1 is the modulation of membrane potential to a drifting grating (Priebe et al., 2004). In mouse V1, we also found neurons that modulate to drifting gratings, but the synaptic basis for this modulation appears different from the cat. In cat V1 neurons, asynchronous inhibition and excitation converge to generate the modulation (Hirsch et al., 1998; Anderson et al., 2000; Monier et al., 2003; Priebe and Ferster, 2005). Asynchronous excitation and inhibition, or pushpull, is thought to contribute to the generation of the linear receptive field characteristics that define cortical simple cells in the cat (Hirsch et al., 1998). In contrast, in mouse V1 neurons, we found that excitation and inhibition were more commonly modulated in phase, synchronously (Fig. 7). This difference in the interplay between excitation and inhibition in the mouse suggests that inhibition plays a distinct role in mouse V1 than cat V1. Such synchronous excitation and inhibition may be the result of the large spatial overlap in ON and OFF receptive fields overlap that exists in mouse V1 (Liu et al., 2010).

How does response selectivity emerge in the mouse cerebral cortex? Our results indicate both similarities and differences with other reported mechanisms underlying cortical selectivity in other species. The importance of spike threshold for enhancing selectivity and the shared selectivity of excitation, inhibition, and membrane potential (Creutzfeldt et al., 1974; Ferster, 1986; Douglas and Martin, 1991; Anderson et al., 2000; Martinez et al., 2002; Monier et al., 2003; Mariño et al., 2005) has now been widely documented in cat visual cortex (Anderson et al., 2000; Monier et al., 2003) and rodent auditory (Wehr and Zador, 2003; Tan et al., 2004) and barrel (Higley and Contreras, 2006) cortex. Excitation and inhibition that adapt equally and inhibition that adapts more strongly than excitation have also been observed in cat visual cortex (Pfleger and Bonds, 1995; Ozeki et al., 2009; Haider et al., 2010), and in other sensory cortices (De Ribaupierre et al., 1972; Wehr and Zador, 2005; Higley and Contreras, 2006; Heiss et al., 2008). Unlike what we observed in the mouse, excitation and inhibition that are temporally modulated at the stimulus frequency typically have been reported as asynchronous in cat visual cortex (Anderson et al., 2000; Priebe and Ferster, 2005, 2006), while we have uncovered synchronous modulations in mouse V1.

This distinction is probably explained by a previously reported difference in the conformation of receptive fields in the cat (Borg-Graham et al., 1998; Hirsch et al., 1998) and mouse (Liu et al., 2009, 2010) V1. Cat layer IV spatial receptive fields consist of segregated "on-off" subfields, in which a light or dark spot evokes 
opposite changes in membrane potential. Based on the cat subfield conformation, therefore, drifting sinusoidal gratings should evoke excitation and inhibition whose temporal modulations are out of phase, as observed in cat simple cells (Fig. 7). In contrast to the cat, light and dark spots both evoke simultaneous excitation and inhibition in mouse visual cortex (Liu et al., 2009, 2010). Based on the mouse subfield conformation, therefore, a drifting grating should evoke excitation and inhibition whose temporal modulations are in phase, as we have observed (Fig. 7). Our observations are thus consistent with the reported subfield conformation differences in cats and mice.

Our observations of differences in the relationship between excitation and inhibition in the cat and mouse raise a number of questions about the direct comparisons we have made. First, it is important to note that the laminar functional organization found in the cat visual cortex is distinct from that in mouse visual cortex. Simple cells are primarily found in layers IV and VI of the cat (Hubel and Wiesel, 1962; Martinez et al., 2005), whereas simple cells are primarily found in layers II/III, IV, and VI in the mouse (Niell and Stryker, 2008). Our comparison of simple cell recordings (Fig. 7) is based on mouse records from layer II-IV and cat records from layer IV, which may underlie some of the discrepancies we observe in the timing of excitation and inhibition. Second, different anesthetics were used for each species (pentothal for cat, pentobarbital for mouse). This anesthetic difference is unlikely to be a source of the changes in excitatory and inhibitory timing observed between the cat and mouse as both of these anesthetics are barbiturates. Nonetheless, these barbiturates have varying effects on EEG amplitude (Roesch et al., 1983). Finally we have attempted to limit age as a factor for our recordings by using mature post-critical period animals (Hubel and Wiesel, 1970; Daw et al., 1992; Gordon and Stryker, 1996).

The role of inhibition in generating response selectivity as a component of the cortical computation remains elusive. We did not find evidence for purely lateral inhibition, but the shared selectivity for excitation and inhibition observed here may nonetheless act to sharpen orientation selectivity. Inhibition may also contribute to selectivity by acting as a gain control mechanism (Heeger, 1992; Carandini and Heeger, 1994; Borg-Graham et al., 1998) or by controlling response timing (Wehr and Zador, 2003; Higley and Contreras, 2006). The balance of excitation and inhibition is a major factor governing critical period plasticity (Hensch et al., 1998; Maffei et al., 2006; Yazaki-Sugiyama et al., 2009). Strikingly, the critical period is not just a period in which right and left eye channels are adjusted within visual cortex, but it is also a period in which the right and left eye orientation selectivities are aligned (Wang et al., 2010). Our observations of the matched orientation selectivity of excitation and inhibition therefore place an important constraint on models of adult cortical circuitry and its development.

\section{References}

Adesnik H, Scanziani M (2010) Lateral competition for cortical space by layer-specific horizontal circuits. Nature 464:1155-1160.

Alitto HJ, Dan Y (2010) Function of inhibition in visual cortical processing. Curr Opin Neurobiol 20:340-346.

Anderson JS, Carandini M, Ferster D (2000) Orientation tuning of input conductance, excitation, and inhibition in cat primary visual cortex. J Neurophysiol 84:909-926.

Azouz R, Gray CM (1999) Cellular mechanisms contributing to response variability of cortical neurons in vivo. J Neurosci 19:2209-2223.

Azouz R, Gray CM (2000) Dynamic spike threshold reveals a mechanism for synaptic coincidence detection in cortical neurons in vivo. Proc Natl Acad Sci U S A 97:8110-8115.

Borg-Graham LJ, Monier C, Frégnac Y (1998) Visual input evokes transient and strong shunting inhibition in visual cortical neurons. Nature 393:369-373.

Brainard DH (1997) The psychophysics toolbox. Spat Vis 10:433-436.

Carandini M, Ferster D (2000) Membrane potential and firing rate in cat primary visual cortex. J Neurosci 20:470-484.

Carandini M, Heeger DJ (1994) Summation and division by neurons in primate visual cortex. Science 264:1333-1336.

Carandini M, Heeger DJ, Movshon JA (1997) Linearity and normalization in simple cells of the macaque primary visual cortex. J Neurosci 17:8621-8644.

Carandini M, Heeger DJ, Movshon JA (1998) Linearity and gain control in V1 simple cells. In: Cerebral cortex, Vol 13, Models of cortical circuits (Ulinski PS, Jones EG, Peters A, eds), pp 401-443. New York: Plenum.

Creutzfeldt OD, Kuhnt U, Benevento LA (1974) An intracellular analysis of visual cortical neurones to moving stimuli: responses in a co-operative neuronal network. Exp Brain Res 21:251-274.

Daw NW, Fox K, Sato H, Czepita D (1992) Critical period for monocular deprivation in the cat visual cortex. J Neurophysiol 67:197-202.

De Ribaupierre F, Goldstein MH Jr, Yeni-Komshian G (1972) Cortical coding of repetitive acoustic pulses. Brain Res 48:205-225.

Douglas RJ, Martin KAC (1991) A functional microcircuit for cat visual cortex. J Physiol 440:735-769.

Dräger UC (1975) Receptive fields of single cells and topography in mouse visual cortex. J Comp Neurol 160:269-290.

Ferster D (1986) Orientation selectivity of synaptic potentials in neurons of cat primary visual cortex. J Neurosci 6:1284-1301.

Ferster D, Jagadeesh B (1992) EPSP-IPSP interactions in cat visual cortex studied with in vivo whole-cell patch recording. J Neurosci 12:1262-1274.

Ferster D, Miller KD (2000) Neural mechanisms of orientation selectivity in the visual cortex. Annu Rev Neurosci 23:441-471.

Gao E, DeAngelis GC, Burkhalter A (2010) Parallel input channels to mouse primary visual cortex. J Neurosci 30:5912-5926.

Gardner JL, Anzai A, Ohzawa I, Freeman RD (1999) Linear and nonlinear contributions to orientation tuning of simple cells in the cat's striate cortex. Vis Neurosci 16:1115-1121.

Gillespie DC, Lampl I, Anderson JS, Ferster D (2001) Dynamics of the orientation tuned membrane potential response in cat primary visual cortex. Nat Neurosci 4:1014-1019.

Gonchar Y, Wang Q, Burkhalter A (2007) Multiple distinct subtypes of GABAergic neurons in mouse visual cortex identified by triple immunostaining. Front Neuroanat 1:3

Gordon JA, Stryker MP (1996) Experience-dependent plasticity of binocular responses in the primary visual cortex of the mouse. J Neurosci 16:3274-3286.

Haider B, Duque A, Hasenstaub AR, McCormick DA (2006) Neocortical network activity in vivo is generated through a dynamic balance of excitation and inhibition. J Neurosci 26:4535-4545.

Haider B, Krause MR, Duque A, Yu Y, Touryan J, Mazer JA, McCormick DA (2010) Synaptic and network mechanisms of sparse and reliable visual cortical activity during nonclassical receptive field stimulation. Neuron $65: 107-121$

Hanover JL, Huang ZJ, Tonegawa S, Stryker MP (1999) Brain-derived neurotrophic factor overexpression induces precocious critical period in mouse visual cortex. J Neurosci 19:RC40.

Heeger DJ (1992) Normalization of cell responses in cat striate cortex. Vis Neurosci 9:181-197.

Heiss JE, Katz Y, Ganmor E, Lampl I (2008) Shift in the balance between excitation and inhibition during sensory adaptation of S1 neurons. J Neurosci 28:13320-13330.

Hensch TK, Fagiolini M, Mataga N, Stryker MP, Baekkeskov S, Kash SF (1998) Local GABA circuit control of experience-dependent plasticity in developing visual cortex. Science 282:1504-1508.

Higley MJ, Contreras D (2006) Balanced excitation and inhibition determine spike timing during frequency adaptation. J Neurosci 26:448-457.

Hirsch JA, Martinez LM (2006) Laminar processing in the visual cortical column. Curr Opin Neurobiol 16:377-384.

Hirsch JA, Alonso JM, Reid RC, Martinez LM (1998) Synaptic integration in striate cortical simple cells. J Neurosci 18:9517-9528.

Hubel DH, Wiesel TN (1962) Receptive fields, binocular interaction and functional architecture in the cat's visual cortex. J Physiol 160:106-154.

Hubel DH, Wiesel TN (1970) The period of susceptibility to the physiological effects of unilateral eye closure in kittens. J Physiol 206:419-436. 
Hübener M (2003) Mouse visual cortex. Curr Opin Neurobiol 13:413-420. Jia H, Rochefort NL, Chen X, Konnerth A (2010) Dendritic organization of sensory input to cortical neurons in vivo. Nature 464:1307-1312.

Johnston D, Wu SMS (1995) Foundations of cellular neurophysiology. Cambridge, MA: MIT Press.

Kerlin AM, Andermann ML, Berezovskii VK, Reid RC (2010) Broadly tuned response properties of diverse inhibitory neuron subtypes in mouse visual cortex. Neuron 67:858-871.

Liu BH, Li P, Li YT, Sun YJ, Yanagawa Y, Obata K, Zhang LI, Tao HW (2009) Visual receptive field structure of cortical inhibitory neurons revealed by two-photon imaging guided recording. J Neurosci 29:10520-10532.

Liu BH, Li P, Sun YJ, Li YT, Zhang LI, Tao HW (2010) Intervening inhibition underlies simple-cell receptive field structure in visual cortex. Nat Neurosci 13:89-96.

Maffei A, Nataraj K, Nelson SB, Turrigiano GG (2006) Potentiation of cortical inhibition by visual deprivation. Nature 443:81-84.

Mangini NJ, Pearlman AL (1980) Laminar distribution of receptive field properties in the primary visual cortex of the mouse. J Comp Neurol 193:203-222.

Margrie TW, Brecht M, Sakmann B (2002) In vivo, low-resistance, wholecell recordings from neurons in the anaesthetized and awake mammalian brain. Pflugers Arch 444:491-498.

Mariño J, Schummers J, Lyon DC, Schwabe L, Beck O, Wiesing P, Obermayer K, Sur M (2005) Invariant computations in local cortical networks with balanced excitation and inhibition. Nat Neurosci 8:194-201.

Martinez LM, Alonso JM, Reid RC, Hirsch JA (2002) Laminar processing of stimulus orientation in cat visual cortex. J Physiol 540:321-333.

Martinez LM, Wang Q, Reid RC, Pillai C, Alonso JM, Sommer FT, Hirsch JA (2005) Receptive field structure varies with layer in the primary visual cortex. Nat Neurosci 8:372-379.

Métin C, Godement P, Imbert M (1988) The primary visual cortex in the mouse: receptive field properties and functional organization. Exp Brain Res 69:594-612.

Monier C, Chavane F, Baudot P, Graham LJ, Frégnac Y (2003) Orientation and direction selectivity of synaptic inputs in visual cortical neurons: a diversity of combinations produces spike tuning. Neuron 37:663-680.

Niell CM, Stryker MP (2008) Highly selective receptive fields in mouse visual cortex. J Neurosci 28:7520-7536.

Ozeki H, Finn IM, Schaffer ES, Miller KD, Ferster D (2009) Inhibitory stabilization of the cortical network underlies visual surround suppression. Neuron 62:578-592.

Pei X, Volgushev M, Vidyasagar TR, Creutzfeldt OD (1991) Whole cell recording and conductance measurements in cat visual cortex in-vivo. Neuroreport 2:485-488.

Pelli DG (1997) The VideoToolbox software for visual psychophysics: transforming numbers into movies. Spat Vis 10:437-442.

Pfleger B, Bonds AB (1995) Dynamic differentiation of GABAA-sensitive influences on orientation selectivity of complex cells in the cat striate cortex. Exp Brain Res 104:81-88.

Priebe NJ, Ferster D (2005) Direction selectivity of excitation and inhibition in simple cells of the cat primary visual cortex. Neuron 45:133-145.

Priebe NJ, Ferster D (2006) Mechanisms underlying cross-orientation suppression in cat visual cortex. Nat Neurosci 9:552-561.
Priebe NJ, Ferster D (2008) Inhibition, spike threshold, and stimulus selectivity in primary visual cortex. Neuron 57:482-497.

Priebe NJ, Mechler F, Carandini M, Ferster D (2004) The contribution of spike threshold to the dichotomy of cortical simple and complex cells. Nat Neurosci 7:1113-1122.

Roesch C, Haselby KA, Paradise RR, Krishna G, Dierdorf S, Wolfe TM, Rao CC (1983) Comparison of cardiovascular effects of thiopental and pentobarbital at equivalent levels of CNS depression. Anesth Analg 62:749753.

Runyan CA, Schummers J, Van Wart A, Kuhlman SJ, Wilson NR, Huang ZJ, Sur M (2010) Response features of parvalbumin-expressing interneurons suggest precise roles for subtypes of inhibition in visual cortex. Neuron 67:847-857.

Shapley R, Hawken M, Ringach DL (2003) Dynamics of orientation selectivity in the primary visual cortex and the importance of cortical inhibition. Neuron 38:689-699.

Sohya K, Kameyama K, Yanagawa Y, Obata K, Tsumoto T (2007) GABAergic neurons are less selective to stimulus orientation than excitatory neurons in layer II/III of visual cortex, as revealed by in vivo functional $\mathrm{Ca}^{2+}$ imaging in transgenic mice. J Neurosci 27:2145-2149.

Sompolinsky H, Shapley R (1997) New perspectives on the mechanisms for orientation selectivity. Curr Opin Neurobiol 7:514-522.

Swindale NV (1998) Orientation tuning curves: empirical description and estimation of parameters. Biol Cybern 78:45-56.

Tan AY, Wehr M (2009) Balanced tone-evoked synaptic excitation and inhibition in mouse auditory cortex. Neuroscience 163:1302-1315.

Tan AY, Zhang LI, Merzenich MM, Schreiner CE (2004) Tone-evoked excitatory and inhibitory synaptic conductances of primary auditory cortex neurons. J Neurophysiol 92:630-643.

Tsodyks MV, Skaggs WE, Sejnowski TJ, McNaughton BL (1997) Paradoxical effects of external modulation of inhibitory interneurons. J Neurosci 17:4382-4388.

Wang BS, Sarnaik R, Cang J (2010) Critical period plasticity matches binocular orientation preference in the visual cortex. Neuron 65:246-256.

Wehr M, Zador AM (2003) Balanced inhibition underlies tuning and sharpens spike timing in auditory cortex. Nature 426:442-446.

Wehr M, Zador AM (2005) Synaptic mechanisms of forward suppression in rat auditory cortex. Neuron 47:437-445.

Wilent WB, Contreras D (2005a) Dynamics of excitation and inhibition underlying stimulus selectivity in rat somatosensory cortex. Nat Neurosci 8:1364-1370.

Wilent WB, Contreras D (2005b) Stimulus-dependent changes in spike threshold enhance feature selectivity in rat barrel cortex neurons. J Neurosci 25:2983-2991.

Yazaki-Sugiyama Y, Kang S, Câteau H, Fukai T, Hensch TK (2009) Bidirectional plasticity in fast-spiking GABA circuits by visual experience. Nature 462:218-221.

Yu Y, Shu Y, McCormick DA (2008) Cortical action potential backpropagation explains spike threshold variability and rapid-onset kinetics. J Neurosci 28:7260-7272.

Zariwala HA, Madisen L, Ahrens KF, Bernard A, Lein ES, Jones AR, Zeng H (2011) Visual tuning properties of genetically identified layer $2 / 3$ neuronal types in the primary visual cortex of cre-transgenic mice. Front Syst Neurosci 4:162, 2011 Jan 13 